\title{
Experiências das mulheres quanto às suas trajetórias até o diagnóstico de endometriose
}

\author{
Experiences of women regarding their pathways to the diagnosis of endometriosis \\ Experiencias de mujeres en cuanto a sus trayectorias hasta el diagnóstico de endometriosis
}

\author{
Carla Marins Silva ${ }^{1}$ (1) \\ Camilla Freitas da Cunha ${ }^{2}$ (1) \\ Karoline Rangel Neves ${ }^{3}$ (D) \\ Victor Hugo Alves Mascarenhas ${ }^{4}$ (1) \\ Adriana Caroci-Becker ${ }^{4,5}$ (D)
}

1. Universidade de São Paulo, Escola de

Enfermagem. São Paulo, SP, Brasil.

2. Secretaria Municipal de Saúde do Rio de Janeiro. Rio de Janeiro, RJ, Brasil.

3. Fundação Oswaldo Cruz, Instituto Fernandes Figueira, Rio de Janeiro, RJ, Brasil.

4. Universidade de São Paulo, Escola de Enfermagem, Programa de Pós-Graduação em Enfermagem. São Paulo, SP, Brasil.

5. Universidade de São Paulo, Escola de Artes, Ciências e Humanidades. São Paulo, SP, Brasil.

Autora Correspondente:

Carla Marins Silva

E-mail: carlamarins@usp.br

Recebido em 10/09/2020.

Aprovado em 01/02/2021.

\section{Resumo}

Objetivo: descrever as experiências das mulheres sobre as suas trajetórias desde o início dos sintomas até o diagnóstico da endometriose. Método: pesquisa descritiva, qualitativa, realizada com dez mulheres com diagnóstico de endometriose no município do Rio de Janeiro (RJ), Brasil. Coletaram-se entrevistas semiestruturadas áudio gravadas e posteriormente submetidas à Análise de Conteúdo por meio do software Atlas.ti 8. Resultados: sem o diagnóstico de endometriose, as mulheres vivenciam sintomas fortes desde a menarca. Essa situação repercute negativamente em diferentes esferas da vida, inclusive pela desvalorização de suas queixas em seus círculos de convivência. Assim, entende-se a importância da rede de apoio perante essa situação. Diante desse contexto, as mulheres peregrinam por diversos profissionais até o diagnóstico definitivo. Considerações finais e implicações para a prática: as trajetórias dessas mulheres são marcadas pela desvalorização de suas queixas por profissionais de saúde e pessoas próximas, pela naturalização da dor feminina e pela dificuldade em estabelecer um diagnóstico diferencial. No entanto, a capacidade individual de reconhecer a presença de uma patologia, o conhecimento sobre a endometriose e a experiência do profissional facilitaram o diagnóstico. No contexto da assistência de enfermagem, entender essa trajetória pode promover a escuta ativa, melhor valorização das queixas, avaliação clínica e o encaminhamento para o diagnóstico precoce.

Palavras-chave: Endometriose; Diagnóstico Clínico; Dor Pélvica; Saúde da Mulher; Ginecologia.

\section{Abstract}

Objectives: to describe the experiences of women on their trajectories from the beginning of symptoms to the diagnosis of endometriosis. Method: descriptive, qualitative research, conducted with ten women diagnosed with endometriosis in the city of Rio de Janeiro (RJ), Brazil. Audio recorded semi-structured interviews were collected and later submitted to Content Analysis using Atlas.ti 8 software. Results: without the diagnosis of endometriosis, women experience strong symptoms from the menarche. This situation has a negative impact on different spheres of life, including the devaluation of their complaints in their circles of coexistence. Thus, the importance of the support network in this situation is understood. Faced with this context, women wander through various professionals until the definitive diagnosis. Final considerations and implications for practice: the trajectories of these women are marked by the devaluation of their complaints by health professionals and people close to them, by the naturalization of female pain and by the difficulty in establishing a differential diagnosis. However, the individual's ability to recognize the presence of a pathology, the knowledge about endometriosis and the professional's experience facilitated the diagnosis. In the context of nursing care, understanding this trajectory can promote active listening, better appreciation of complaints, clinical assessment and referral to early diagnosis.

Keywords: Endometriosis; Clinical Diagnosis; Pelvic Pain; Women's Health; Gynecology.

\section{Resumen}

Objetivo: describir las vivencias de las mujeres en sus trayectorias desde el inicio de los síntomas hasta el diagnóstico de endometriosis. Método: investigación descriptiva cualitativa realizada con diez mujeres diagnosticadas con endometriosis en la ciudad de Río de Janeiro (RJ), Brasil. Se recogieron entrevistas semiestructuradas grabadas en audio y posteriormente se sometieron a Análisis de Contenido utilizando el software Atlas.ti 8. Resultados: sin el diagnóstico de endometriosis, las mujeres experimentan síntomas fuertes desde la menarquia. Esta situación tiene un impacto negativo en diferentes ámbitos de la vida, incluso por la devaluación de sus quejas en sus círculos de convivencia. Así, se comprende la importancia de la red de apoyo en esta situación. Ante este contexto, las mujeres deambulan por diferentes profesionales hasta el diagnóstico definitivo. Consideraciones finales e implicaciones para la práctica: las trayectorias de estas mujeres están marcadas por la devaluación de sus quejas por parte de los profesionales de la salud y personas cercanas, por la naturalización del dolor femenino y por la dificultad para establecer un diagnóstico diferencial. Sin embargo, la capacidad del individuo para reconocer la presencia de una patología, el conocimiento sobre la endometriosis y la experiencia del profesional facilitaron el diagnóstico. En el contexto del cuidado de enfermería, comprender esta trayectoria puede promover la escucha activa, mejor valoración de las quejas, evaluación clínica y la derivación para diagnóstico precoz.

Palabras clave: Endometriosis; Diagnóstico Clínico; Dolor Pélvico; Salud de la Mujer; Ginecología. 


\section{INTRODUÇÃO}

A endometriose é uma afecção ginecológica, decorrente da presença de tecido endometrial ectópico, que afeta entre cinco e $15 \%$ de mulheres em idade reprodutiva no mundo. ${ }^{1-3}$ No Brasil, as estimativas de prevalência da endometriose apontam que cerca de sete milhões de brasileiras sofrem com essa patologia. ${ }^{4}$ Apesar disso, acredita-se que os dados epidemiológicos informados ainda são inconclusivos. A dificuldade em levantar informações fidedignas deve-se principalmente à dificuldade de acesso ao diagnóstico definitivo, cirúrgico, e à banalização dos sintomas femininos pela sociedade, bem como pelos profissionais de saúde. 5,6

Em nível nacional, a doença é relativamente desconhecida pela população em geral. Os pacientes frequentemente recebem seus diagnósticos tardiamente, apesar de a maioria desenvolver os sintomas iniciais durante a adolescência. Sem um diagnóstico definitivo para as suas queixas, a maioria das mulheres apresenta dificuldade para relatar suas irregularidades menstruais ou procura ocultá-las para evitar uma estigmatização devido aos traços culturais, que tendem a naturalizar a dor durante o período menstrual. ${ }^{5}$

A análise do tempo médio de diagnóstico da endometriose em países desenvolvidos e em desenvolvimento identificou um atraso de 6,7 anos entre o início dos sintomas até o diagnóstico definitivo. Ressalta-se que as mulheres procuraram consultas médicas em um tempo médio de até um ano após os primeiros sintomas e aquelas que utilizaram os serviços de saúde pública apresentaram maior atraso, em média, de 8,5 anos, quando comparadas àquelas que fizeram uso de serviços privados, com média de 5,5 anos. ${ }^{7}$ Além disso, a caracterização sociodemográfica dessas mulheres permitiu identificar uma predominância da população branca e com alto grau de escolaridade, o que contribui para compreender que o acesso ao diagnóstico de endometriose pode ser um processo bastante excludente. ${ }^{8}$

A demora quanto ao diagnóstico deve ser tratada como um ponto de intensa preocupação, uma vez que pode resultar em um tratamento tardio ou inadequado, bem como desenvolver desfechos mais graves, como um maior risco de infertilidade e lesões em órgãos subjacentes. Os sinais e os sintomas da endometriose, quando não gerenciados, afetam diretamente a qualidade de vida das mulheres e contribuem para a perda de produtividade ou a incapacidade de desempenhar atividade laboral. .,10 $^{10}$

Desse modo, este artigo justifica-se pela necessidade de preencher uma lacuna na literatura nacional com relação à trajetória até o diagnóstico da endometriose sob a ótica das mulheres. Entende-se que a temática permeia questões que vão além do modelo biomédico, como a construção cultural do feminino e a relação profissional-paciente. Por isso, esses relatos ganham relevância para explicar a negligência referente a essa afecção. No contexto da assistência de Enfermagem, as experiências das mulheres podem estimular os profissionais a prestar um cuidado mais humanizado e a promover a escuta ativa, a valorização das queixas da paciente, a avaliação clínica e o encaminhamento para o diagnóstico precoce.

Diante disso, a questão norteadora é: "Qual a experiência de mulheres sobre as suas trajetórias desde o surgimento dos sintomas até o diagnóstico definitivo de endometriose?". Objetivase descrever as experiências das mulheres sobre as trajetórias desde o início dos sintomas até o diagnóstico da endometriose.

\section{MÉTODO}

Trata-se de uma pesquisa descritiva com abordagem qualitativa. A escolha por esse método surgiu para as descrições sobre as experiências das participantes, sendo o fenômeno do estudo. Busca-se descrever os achados provenientes dos significados, motivos, aspirações, crenças, valores e atitudes dessas mulheres. ${ }^{11}$

Recrutaram-se mulheres, de forma intencional, com base nos critérios de inclusão: ter 18 anos ou mais de idade com diagnóstico de endometriose; ser residente no município do Rio de Janeiro; participar de grupos fechados de uma rede social da internet e aceitar participar do estudo. Estabeleceu-se como critério de exclusão: ter endometriose, mas ser assintomática.

A interlocução com os atores sociais na coleta dos depoimentos desta pesquisa permitiu uma aproximação com o fenômeno estudado. Apesar da homogeneidade da amostra, ela reflete a subjetividade e as relações interpessoais desse grupo específico, corroborando o perfil socioeconômico identificado nos contextos nacional e internacional. ${ }^{8}$ Dessa forma, o ponto de saturação ocorreu tanto pelo volume de dados quanto pelo nível de detalhamento e aprofundamento sobre as trajetórias dessas mulheres, demonstrando as diversas dimensões do objeto de pesquisa. ${ }^{12}$

A captação de potenciais participantes do estudo ocorreu por meio de uma rede social de acesso via on-line. Inicialmente, realizou-se uma busca inicial por grupos fechados na rede social utilizando a palavra "endometriose"; em seguida, selecionaram-se apenas os grupos formados por mulheres diagnosticadas com endometriose, observando o teor de postagens ou os comentários que abordassem a temática. Após o processo de seleção do grupo, solicitou-se a autorização para participar como membro do grupo fechado selecionado.

Posteriormente, ocorreu a etapa de recrutamento das participantes e os pesquisadores criaram uma postagem no grupo em que foram esclarecidos a justificativa da pesquisa, os objetivos e os critérios de inclusão e exclusão para participar do estudo. À medida que as possíveis candidatas a participar do estudo manifestaram interesse em contribuir, os pesquisadores entraram em contato individualmente por mensagem privada, ainda na rede social, e marcaram um posterior encontro para a realização de entrevistas. Vale destacar que nenhuma participante se recusou a participar ou interrompeu a sua participação na pesquisa.

O período de coleta de dados ocorreu entre dezembro de 2016 e janeiro de 2017. As entrevistas foram realizadas de forma individual e presencial em locais públicos escolhidos pelas entrevistadas com o intuito de promover o acolhimento e a segurança à participante. Os ambientes selecionados foram locais distantes do trânsito de pessoas para garantir o máximo de privacidade das entrevistadas. Os diálogos foram gravados em áudio, por meio de um gravador digital, e transcritos literalmente para posterior análise. O tempo médio da entrevista foi de 40 minutos. As transcrições foram disponibilizadas às respectivas 
entrevistadas para fins de verificação, mas não houve qualquer solicitação de alteração de conteúdo.

$O$ instrumento de coleta de dados continha um roteiro de entrevista semiestruturada. As questões disparadoras foram "Conte-me como foi a sua vivência no início dos sintomas. Como chegou ao diagnóstico de endometriose?". Tais questionamentos foram acompanhados de tópicos, introduzidos ao longo da entrevista, com o intuito de conduzir a discussão e direcionar as questões e as informações não esclarecidas, como os sintomas, as repercussões na vida pessoal, as repercussões no trabalho, a peregrinação até o diagnóstico, os custos, os exames, as consultas e a desvalorização das queixas.

Antes de responderem aos questionamentos, todas as participantes foram informadas sobre os objetivos da pesquisa e consentiram participar por meio da assinatura do Termo de Consentimento Livre e Esclarecido (TCLE).

As análises do conteúdo das entrevistas foram conduzidas usando o software de análises qualitativas Atlas.ti 8 e, para o tratamento de dados, utilizou-se o levantamento de conteúdo temático/categorial de Bardin. ${ }^{13}$ Com base na leitura e na interpretação das mensagens, ocorreu a compreensão dos significados, seguida pelo processo de codificação e finalizada pela inferência dos dados.

A abordagem de codificação foi realizada por dois codificadores independentes e ocorreu em três etapas: identificação de códigos e Unidades de Registro (UR), trechos das entrevistas destacados com diferentes cores, por meio do método indutivo de análise de dados; agrupamento das UR para a elaboração dos temas; formação de categorias e subcategorias.

As discrepâncias que surgiram foram resolvidas por consenso entre ambos os codificadores. Os resultados dessa etapa foram disponibilizados às participantes do estudo a fim de avaliar a confiabilidade, a credibilidade e a generalização dos achados.

As inferências ocorreram com base nos agrupamentos semânticos. Categorias e subcategorias foram descritas por textos explicativos, ilustradas com as árvores de codificação elaboradas no software e exemplificadas com as UR para cada subcategoria correspondente. Por fim, a discussão do conteúdo analisado nas categorias foi realizada com base na literatura científica com o intuito de validar os achados deste estudo.

Esta pesquisa foi aprovada pelo Comitê de Ética em Pesquisa da Universidade do Estado do Rio de Janeiro (CAAE: 1.650.425/2016). Todos os preceitos éticos da Resolução ํㅜ 466/2012, do Conselho Nacional de Saúde, foram respeitados, garantindo o sigilo e a integridade das participantes. Para manter o anonimato nas falas, adotou-se a nomeação com a letra inicial "E" de endometriose, seguida pelo número de ordem de realização da entrevista, por exemplo, “E1, E2, E3...".

\section{RESULTADOS}

Entrevistaram-se dez mulheres no estudo com faixa etária entre 18 e 38 anos de idade e com situação conjugal majoritariamente composta por casadas $(n=7)$. Houve uma predominância $(n=7)$ da população branca e a prevalência de alto grau de instrução, em que cinco possuíam o Ensino Superior completo, seguidas de duas com Ensino Superior incompleto e duas com Ensino Médio completo.

A média de tempo entre o início dos sintomas e a data do diagnóstico foi de 6,5 anos. A maioria das mulheres $(n=6)$ referiu a dor pélvica como queixa principal; em seguida, houve queixas de dismenorreia $(n=4)$, dispareunia $(n=3)$, infertilidade $(n=3)$ e fluxo intenso $(n=2)$.

No total, identificaram-se 104 unidades de registros nas transcrições das entrevistas, que foram agrupadas em duas categorias e seis subcategorias. Sistematizaram-se as vivências das mulheres com relação às trajetórias ao diagnóstico de endometriose nas categorias temáticas: "Sofrendo com os sintomas sem o diagnóstico de endometriose" e "Peregrinando até o diagnóstico de endometriose" (Figura 1).

\section{Sofrendo com os sintomas sem o diagnóstico de endometriose}

Nesta categoria, identificou-se um total de 50 unidades de registro e três subcategorias. $O$ sofrimento inicial de quem convive com os sintomas de endometriose perpassa por três importantes condições: os fortes sintomas vivenciados desde a primeira menstruação, as repercussões negativas em diferentes esferas da vida das mulheres e a importância de uma rede de apoio perante essa situação.

\section{Sintomas fortes desde a primeira menstruação, denominada menarca}

As participantes relataram que, desde a menarca, sofriam com dores acima do limiar aceitável, nunca tiveram um ciclo menstrual sem dor, referente a cólicas fortes, incapacitantes e presentes até mesmo fora do período menstrual. Acreditam que a endometriose começou a se desenvolver a partir da primeira menstruação.

Então, desde a primeira menstruação, foi com dor. Eu nunca, na minha vida, lembro de ter tido uma menstruação, um ciclo sem dor. (E10)

Os sintomas físicos comumente mencionados foram dor pélvica, dismenorreia, dispareunia e infertilidade. Também descreveram os sintomas associados à fisiologia intestinal, como diarreia, prisão de ventre, dor e sangramento ao evacuar. Além disso, disfunções ginecológicas como a irregularidade menstrual e o fluxo menstrual intenso.

Eu falava com ele que sentia dor, muita dor na penetração e muita cólica. (E5)

Dornas costas, dornas pernas, dor para evacuar [...] um fluxo absurdo, longo e intenso [...] com muito vômito. Só que isso não passou. (E10)

Além dos sintomas físicos, as mulheres também apresentaram sintomas psicológicos. As experiências psicológicas mais discutidas incluíram estresse e angústia em relação ao contexto 


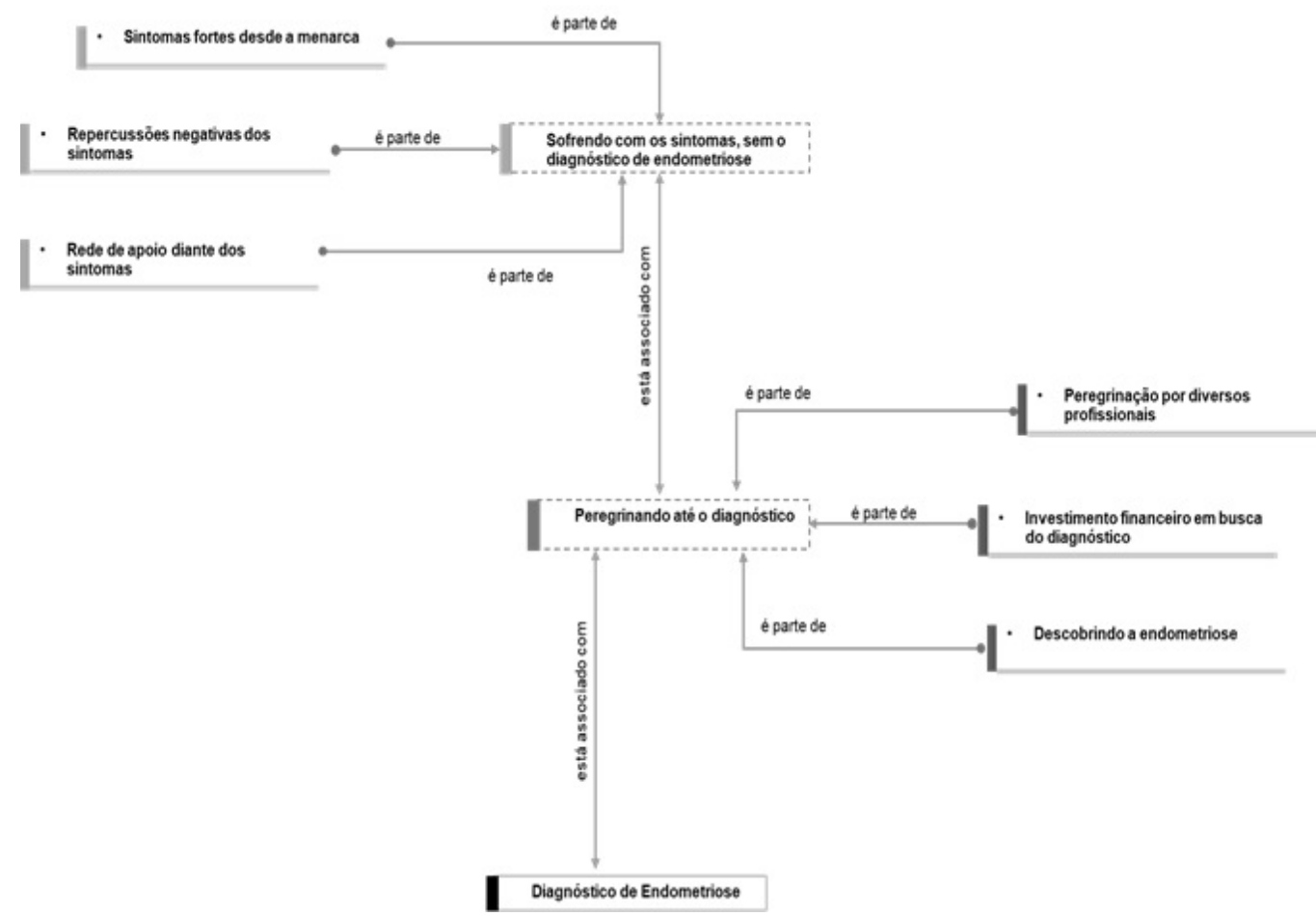

Figura 1. Árvore de codificação das trajetórias de mulheres até o diagnóstico de endometriose elaborada no software Atlas.ti 8. Fonte: Atlas.ti.8.

em que estavam inseridas, caracterizados como "dor na alma" e "dor emocional".

Então, além da dor física, a dor emocional também, né? (E2)

É complicado, né? Não é uma dor só física, é uma dor na alma... É muito ruim... (E7)

\section{Repercussões negativas dos sintomas.}

Muitas mulheres transmitiram a sensação de que os seus círculos de convivência desvalorizavam as suas queixas. As participantes percebiam julgamentos pessoais sobre os seus sintomas vistos como "bobagem", "frescura" ou "coisa de mulherzinha". A percepção de que seus sintomas eram ignorados revela a falta de empatia por parte de quem deveria acolhê-las. A ausência real ou percebida de apoio social repercute diretamente no estado emocional dessas mulheres.

E a pessoa, no trabalho, diz que entende, mas não entende: "Porque ter cólica é frescura" [...] então, assim é complicado, porque as pessoas ficam: "não, eu super entendo", mas não entendem nada. (E1)

Acha que é bobeira, frescura, mas eu não conseguia nem me levantar da cama, dava vontade de desmaiar [...] aí, sempre ouvia gracinha, né? Porque as pessoas não entendem o que é... (E8)
Durante a conversa, revelou-se que os sintomas da doença pioravam com o passar do tempo e que os intervalos entre as crises de dor eram cada vez menores. Não somente a doença agravava a condição de saúde das mulheres, mas também despertava um sentimento de impotência ao perceber que o uso de analgésicos e antiespasmódicos proporcionava um alívio nas dores relacionadas à endometriose, mas não era suficiente para eliminar o sintoma ou curar a doença. As experiências compartilhadas mencionam que os fármacos deixavam de fazer o efeito desejado e, por isso, periodicamente, havia troca de medicação.

Nunca passava, por mais remédio que eu tomasse [...] demorava muito tempo. (E6)

Só foi piorando [...] fora remédio para dor... porque um [antiespasmódico e analgésico] não dá para nada... Nenhum remédio cura... Nenhum remédio tira a dor...nenhum alivia [...]não aliviava nada... Tudo história de fantasia. (E7)

Com a ineficácia dos analgésicos, as participantes iam frequentemente ao pronto-socorro, durante as crises, onde faziam o uso de fármacos por via intravenosa para o alívio imediato da dor. Apesar da concepção social e pessoal de que os sintomas eram considerados normais, a baixa efetividade dos medicamentos e a frequente procura por serviços de urgência apontavam para o fato de que o quadro não era fisiológico. 
Parava em pronto-socorro porque tinha vez que o remédio já não fazia efeito [...] todo mês, era pronto-socorro para tomar injeção na veia [...] aí, na adolescência mesmo é que foi piorando, né? Todo mês era pronto-socorro, isso não podia ser normal. (E4)

Além disso, dentre as histórias compartilhadas, observou-se que a endometriose repercute também no âmbito laboral, quando reduz o desempenho profissional e as suas percepções dos sintomas são desvalorizadas. As frequentes queixas álgicas parecem ser irrelevantes em meio à necessidade de produção do mercado de trabalho. Como resposta a esse contexto, percebe-se o absenteísmo ou o presenteísmo devido à falta de condições físicas e emocionais para trabalhar.

Porque, por mais que você justifique que você passa mal, que você desmaia, a pessoa não está preocupada. Ela quer saber se, no final, o número vai estar batendo e se vai ter feito a quantidade que o contrato diz. Então, assim, isso é muito difícil. (E1)

Mas, já fui sim (para o trabalho) me arrastando [...] aí, eu vou dopada de remédio, mas vou [...] já fui, muitas vezes, pedindo para morrer no metrô com muita cólica... Me esvaindo em sangue. [...] Eu sempre vou porque eu acho que vou ser mal-vista, entendeu? (E2)

Algumas mulheres transmitiram a ideia de que os sintomas têm impacto sobre a vida social quando as impedem de realizar atividades prazerosas ou afetam seus relacionamentos amorosos.

Mas, eu vi que estava ficando só ruim e estava me afastando do meu esposo. (E5)

Eu até fui viajar com o meu esposo, para casa do meu irmão, lá em Rio das Ostras. No meio de uma festa, eu tive que ir embora porque eu passei muito mal e foi de repente. (E7)

\section{Rede de apoio diante dos sintomas}

Apesar das queixas sobre a desvalorização dos intensos sintomas de endometriose por pessoas do seu grupo social próximo, reconheceram um suporte social específico, principalmente por parte das pessoas com mais vínculo afetivo. Em diferentes graus e formas, a família, os companheiros e as amigas foram mencionados como a principal rede de apoio em meio aos sintomas.

Mas, eles sempre me apoiaram muito: "Vamos procurar outro médico"; "Não gostou? vamos procurar outro"; "Faz exame". Na questão familiar, tudo tranquilo, graças a Deus. (E1)

Então, eu tenho um maridão... Eu tenho um marido maravilhoso e ele me ajuda... ele é muito compreensivo. (E7)
Teve um dia que eu não aguentei. Aí, minhas amigas me orientaram: "Você deve estar com endometriose" (...) aí, eu fui ao médico. (E3)

\section{Peregrinando até o diagnóstico}

Nesta categoria, identificou-se um total de 54 unidades de registro e três subcategorias. As trajetórias das mulheres até obter o diagnóstico de endometriose possuem pontos favoráveis e dificultadores que foram levantados na análise dos relatos.

\section{Peregrinação por diversos profissionais}

$\mathrm{Na}$ busca por profissionais que respondessem às suas demandas, muitas mulheres perceberam que alguns não valorizavam as suas queixas como deveriam. Esse problema é interpretado como descaso ou falta de interesse por parte dos profissionais, conforme se observa nas unidades de registro analisadas.

Ah, naquela época, era cólica menstrual... só isso que falavam... Toma ali um [antiespasmódico] na veia e vai para casa... Era só isso que eu ouvia: "ah, cólica menstrual". (E4)

Foi onde eu falei: é o descaso...é falta de interesse dos médicos [...] Então, muitos não tinham paciência... então, logo cortavam: "Ah, essa daí é uma cólica, coloca uma bolsa de água quente".(E7)

Apesar de haver uma variedade de exames para identificar a endometriose, existem muitas incertezas no diagnóstico. A dificuldade em realizar um diagnóstico diferencial foi um dos problemas apontados pelas participantes em que, muitas vezes, os seus sintomas eram associados a infecções sexualmente transmissíveis ou a qualquer outro tipo de infecção.

Toda vez que você vai ao ginecologista, ele sempre passa antibiótico, pomada. É sempre a mesma coisa. [...] você está com uma ferida no útero, "ah, é inflamação". (E8)

$E$, depois disso, eu comecei a sentir dor na relação e procurei a ginecologista e, a princípio, ela investigou DST. Então, fiz todos os exames de clamídia, gonorreia, exames que o plano de saúde não cobria [...] porque a médica batia na tecla da clamídia, principalmente. (E9)

\section{Investimento financeiro em busca do diagnóstico}

As dificuldades dos profissionais em identificar o quadro clínico da endometriose desencadeiam custos financeiros para as pacientes. Gastos com especialistas da rede privada e diversos exames solicitados foram observados nos relatos das mulheres.

De acordo com essas experiências, os planos de saúde são alternativas que ajudam a diminuir os custos com a doença, e o atendimento pelo serviço púbico de saúde foi definido como "demorado" e de "difícil acesso". 
Já gastei acho que uns três mil reais em médico... Por cima [...] e para ter um diagnóstico decente, tem que ir a um médico particular. (E2)

Não, os custos são sempre; com o plano de saúde não é tanto porque os exames são caros e o plano cobre. (E3)

E demorou porque, como eu te falei, onegócio de hospital público... Mandavam fazer exames que não tinham nada a ver. (E7)

Os gastos com alguns exames pareceram ser inviáveis quando, apesar de acessar diversas tecnologias, não foi possível identificar sinais da doença. No entanto, os exames funcionaram como peça-chave para confirmar o diagnóstico de endometriose.

Fazia exame, não acusava nada. (E6)

A primeira coisa foi uma ultra e ele colocou como indicação que poderia ser endometriose. Mas, eu teria que fazer um exame mais especifico, que foi a ressonância com preparo intestinal. O que indicou mesmo foi a ressonância e o CA-125 que, na época, se eu não me engano, o meu estava quinhentos e pouco, bem alto. (E8)

\section{Enfim, descobrindo a endometriose}

Nas trajetórias das mulheres, para conseguir o diagnóstico definitivo, alguns pontos foram extremamente importantes para esses desfechos positivos. Algumas mulheres discutiram sobre infertilidade, citando a dificuldade de concepção como um dos fatores que permitiram a descoberta da endometriose, pois, a partir dessa condição, procuraram se informar e buscar profissionais especializados.

Eu falei: "Ah, mas eu quero engravidar". Mas, não engravidava [...] aí, meu marido fez o espermograma e tudo bem... Estava tudo certo e eu nada de vir a criança [...] foi em outubro de 2014 que eu comecei a querer ser mãe. O diagnóstico foi agosto de 2015. (E2)

Diante da complexidade em estabelecer o diagnóstico diferencial e da dificuldade de alguns profissionais em reconhecer o quadro clínico, destaca-se que a experiência e a competência profissional foram fatores primordiais para poder identificar e conduzir a endometriose.

Mas, foi só com ajuda desse, o experiente... Dos 13 aos 25 anos, ninguém descobria. (E7)

Tem um centro de endometriose em São Paulo, minha mãe marcou e nós fomos [...] lá, eu comentei o que estava passando, sofrendo. Aí, ela fez todo o inquérito de endometriose e tudo batia. Aí, ela falou para mim: "eu acho que você tem endometriose". (E10)
Dentre os principais fatores que possibilitaram findar essa trajetória, ressalta-se que a capacidade individual das mulheres em reconhecer a presença de uma patologia instaurada é o mais importante. Assim, laudos de exames equivocados e profissionais que desvalorizam suas queixas sintomáticas não são impeditivos da busca pelo diagnóstico da doença.

Eu sabia que não era normal essa dor esquisita, eu sabia que não era normal e continuei buscando. (E3)

Eu vou procurar, isso não é da minha cabeça. Aí, eu comecei a procurar o tratamento a partir daí. (E5)

E aí eles diziam que era ovulação dolorosa, fazia uma ultra: "Ah, é ovulação dolorosa". Aí, eu falava: "Gente, mas ninguém sente a mesma dor que eu sinto... tinha alguma coisa diferente."[...] mas, eu passei sete anos dizendo que eu sentia muita dor... vendo que não era normal. (E10)

\section{DISCUSSÃO}

Neste estudo, a caracterização sociodemográfica das mulheres entrevistadas com o diagnóstico de endometriose permitiu verificar que houve uma predominância da população branca e com alto grau de escolaridade, sendo que a maior parte da população no Brasil é de mulheres pardas e negras. ${ }^{14}$ Esses achados são semelhantes aos de um estudo de revisão sistemática ${ }^{2} e$ pode-se sugerir que as mulheres não brancas e com menos grau de escolaridade podem ter mais dificuldade em ter acesso ao diagnóstico de endometriose do que as mulheres brancas.

Em pesquisa que objetivou avaliar a experiência de 4.334 mulheres que tiveram o diagnóstico de endometriose por meio de uma intervenção cirúrgica, os resultados identificaram que elas eram predominantemente de cor branca (3.696 - 94,4\%). Outras pesquisas realizadas no Brasil também verificaram que algumas intervenções cirúrgicas, como a cesariana, são muito mais frequentes entre as mulheres brancas do que entre as não brancas e menos frequentes entre as usuárias do setor público e com menor grau de escolaridade. ${ }^{8,14-16}$

Os resultados deste e de outros estudos possibilitaram compreender como é a experiência de mulheres que têm endometriose, mas enfrentam um tortuoso caminho até obter o diagnóstico definitivo. Essa dificuldade em estabelecer um diagnóstico produz impacto negativo em suas vidas, uma vez que, até consegui-lo, tendem a conviver com os sintomas sem um tratamento adequado. 2,6,8,17

Embora as participantes tenham relacionado a origem de suas queixas ao início da adolescência, essa experiência não foi um fator suficiente para levantar suspeitas quanto à presença da endometriose. Segundo a American College Obstetricians and Gynecologists, a prevalência de endometriose em adolescentes aindaé desconhecida. No entanto, dois terços das adolescentes que referiram sofrer com a dor pélvica crônica ou a dismenorreia, sem responder corretamente ao uso de anti-inflamatórios não esteroidais ou à terapia hormonal, foram futuramente diagnosticadas com endometriose por meio da laparoscopia. ${ }^{18}$ Assim sendo, os sintomas manifestados no princípio 
da idade fértil devem ser mais bem observados e valorizados quando se objetiva um diagnóstico precoce e adequado.

Conforme as recomendações do National Institute for Health and Clinical Excellence (NICE), são consideradas, como casos suspeitos de endometriose, mulheres, incluindo jovens com idade igual ou inferior a 17 anos, que apresentem um ou mais dos seguintes sinais e sintomas: dor pélvica crônica; dismenorreia que impeça realizar atividades diárias; dispareunia profunda; sintomas gastrointestinais que ocorram de forma periódica; sintomas cíclicos de disfunção urinária como a disúria e a hematúria; infertilidade associada a uma ou mais das queixas anteriores. ${ }^{3}$ Nesse sentido, observou-se que todas as experiências discutidas neste estudo tratavam-se de quadros clínicos de endometriose em que as participantes apresentaram sinais e sintomas esperados para essa suspeita, desde o princípio da doença, assim como identificado em outros estudos também. . $^{2,6,8,17}$

O impacto psicológico da doença também foi outro ponto levantado pelas mulheres como algo além dos sintomas físicos enfrentados e esses resultados assemelham-se aos de outras pesquisas, permitindo compreender que as repercussões negativas na saúde mental das participantes deveram-se ao aspecto crônico da dor, a diagnósticos incorretos e às opções limitadas de tratamento. A construção cultural influencia a forma como a sociedade encara as queixas álgicas femininas, e a falácia de que a mulher deve estabelecer proximidade identitária com suas sensações dolorosas impede que as queixas características da endometriose sejam consideradas genuínas. Desse modo, as dores do parto e das cólicas menstruais são encaradas como símbolos ideais do gênero feminino e aquelas que não conseguem suportá-las tendem a ser vistas como inferiores ou frágeis. ${ }^{2,6,17}$

Mulheres diagnosticadas com endometriose apresentaram níveis superiores de depressão quando comparadas às mulheres saudáveis. Aquelas que referiam queixas intensas de dor pélvica também apresentavam níveis superiores e estatisticamente significativos em relação às que não relataram esse sintoma..$^{5,19}$

Essa situação é agravada com as crises que tendem a piorar com o tempo, tornando os analgésicos e antiespasmódicos totalmente ineficazes. O tratamento empírico da dor consiste na terapia hormonal combinada com o uso de anti-inflamatórios não esteroidais, sendo uma opção viável enquanto o tratamento cirúrgico não está acessível. As mulheres têm demonstrado interesse em buscar o alívio da dor pelo uso de práticas integrativas e complementares, como a acupuntura, a homeopatia, a meditação e a dieta..$^{20,21}$

Os resultados deste estudo apontam para a procura das mulheres por serviços de urgência para o tratamento dos sintomas da endometriose e isso pode revelar uma falha quanto à organização das redes de atenção primária de saúde. O acesso periódico ao pronto-socorro contribui para o atraso no diagnóstico, uma vez que esses serviços apresentam foco apenas na atenção imediata às queixas e não ao tratamento. A condução adequada das pacientes nesse sistema deveria promover o alívio das queixas álgicas, seguido do encaminhamento à devida especialidade. ${ }^{6}$

Na esfera ocupacional, estudos congêneres ratificaram os achados deste estudo, em que se destacaram o presenteísmo e o absenteísmo do trabalho. O absenteísmo esteve relacionado a situações extremas nas quais se esgotavam as possibilidades de licenças médicas ou condições em que não se recebeu suporte por parte dos empregadores. Em contrapartida, o presenteísmo esteve atrelado a condições nas quais a família dependia unicamente da fonte de renda das mulheres. ${ }^{5,10}$

As mulheres escondem suas próprias condições de seus colegas do sexo masculino. Assim, percebe-se que as questões da fisiologia reprodutiva feminina ainda são consideradas "tabus", tendo em vista que, assim como neste estudo, há uma preocupação com relação às acusações e aos julgamentos dos colegas de trabalho. Muitas vezes, os fortes sintomas são interpretados equivocadamente como pretextos para não desempenhar suas tarefas ou apenas "fraqueza do sexo feminino". ${ }^{22}$

A endometriose pode afetar negativamente a vida social das mulheres, pois os resultados mostraram que as mulheres desta pesquisa referiram sentir dor e desconforto em um nível que as impediu de realizar as atividades diárias. Um estudo de revisão sistemática de métodos qualitativos evidenciou que há uma redução na participação de atividades sociais associada principalmente aos sintomas debilitantes e às questões estruturais como a necessidade de um banheiro disponível e o medo de manifestar os sintomas em público. ${ }^{22}$

O impacto no aspecto social reflete na qualidade de vida das mulheres. Estudos avaliativos identificaram a associação da presença de endometriose com uma avalição negativa quanto ao domínio "funcionamento social". O caráter limitador da doença em suas atividades cotidianas dificulta a realização de suas próprias atividades ou o comparecimento em eventos sociais, o que desenvolve um nível de ansiedade nas pacientes devido à imprevisibilidade dos surgimentos das crises que culmina no sentimento de impotência quanto ao seu estado de saúde e, muitas vezes, à incompreensão por parte do seu ciclo de convivência. ${ }^{17,23}$

O amparo social foi fundamental para o enfrentamento da doença, pois muitas mulheres destacaram receber apoio dos parceiros, amigos e familiares. Uma metassíntese de estudos de países em desenvolvimento evidenciou que a presença ou a ausência do apoio social poderia ditar o enfrentamento quanto às experiências menstruais. Essa influência torna-se importante quando auxilia a concluir atividades, promove o suporte emocional ou até mesmo funciona como fonte de informação. ${ }^{24}$

No que se refere ao investimento financeiro, identificaramse gastos específicos com relação à intensa peregrinação das mulheres por procura de profissionais e à realização de diversos exames, bem como aos problemas de ordem laboral. Na literatura científica, há um consenso nos casos de endometriose para que as mulheres priorizem a realização de um plano de saúde..$^{5,17}$

Um grande estudo transversal, considerado a maior pesquisa multicêntrica prospectiva sobre os efeitos da endometriose realizada até o momento, evidenciou que a banalização das queixas das mulheres pelos profissionais é um problema de ordem mundial. Quando as pacientes peregrinam pelo diagnóstico, são informadas, pelos seus médicos, que os sintomas apresentados são considerados normais, tendo em vista a fisiologia do ciclo menstrual. Resultado 
de como os serviços de saúde incorporam os estereótipos de gênero reflexos de uma visão social deturpada com relação ao feminino. Dessa forma, os sintomas narrados são sistematicamente desvalorizados pelos profissionais e as informações quanto à patologia seguem sem ter a devida divulgação. ${ }^{6,7}$

Algumas atitudes e comportamentos dos profissionais de saúde foram descritos pelas mulheres do estudo atual como problemas. Muitas vezes, não houve interesse, por parte dos profissionais, em identificar os aspectos importantes da dor, como a quantidade, a intensidade, a duração, a natureza ou o impacto. Classificar a dor da endometriose como normal revela uma falta de treinamento ou atualização por parte de alguns profissionais, falta de escuta ativa, tratamento de seus sintomas e devidos encaminhamentos. Compreende-se que essa forma de condução e atendimento não está diretamente relacionada a uma possível imperícia profissional, mas se entende que, apesar de os especialistas conhecerem sobre a patologia, isso não é suficiente para a identificação da doença. Por isso, atribui-se grande parte dos atrasos de diagnóstico à negligência profissional. . 22,25

Uma pesquisa holandesa apontou que a experiência e a competência dos profissionais, a boa relação com os seus pacientes, a orientação e a colaboração profissional foram os principais fatores que contribuíram para a descoberta do diagnóstico precocemente. ${ }^{9}$ Além disso, as diferentes apresentações clínicas da endometriose exigem a presença de um corpo multidisciplinar para elaborar um adequado plano de atenção e diferentes olhares e vertentes do cuidado contribuem para a integralidade do atendimento às mulheres. A construção de protocolos desenvolvidos por diferentes especialidades para o diagnóstico e o tratamento da endometriose também é uma ferramenta que pode contribuir para uma melhor resolutividade na condução clínica. ${ }^{17,23}$

Outro ponto abordado neste estudo é a dificuldade para engravidar como importante na obtenção do diagnóstico de endometriose de forma mais rápida. Esse tipo de associação expressa uma questão de gênero institucionalizada na assistência: o paradigma da necessidade de função reprodutiva feminina em detrimento das demais queixas apresentadas. $\mathrm{O}$ impacto da endometriose na fertilidade pode afetar não apenas o bemestar das pacientes, mas também suas relações pessoais. A possibilidade de haver infertilidade com a doença manifesta sentimentos de preocupação e melancolia nas pacientes, tendo em vista que a construção social da ideia de que a mulher deve ser capaz de conceber uma vida resulta em julgamentos internos e externos em volta do conceito de "mulher de verdade".

A European Society of Human Reproduction and Embryology (ESHRE) recomenda que a sequência preconizada para o diagnóstico de endometriose constitui-se de, inicialmente, considerar caso suspeito com base no histórico, sinais e sintomas; seguidamente, corroborar a suspeita com exame físico e os exames de imagem; finalmente, comprovar a partir da análise histológica de amostras colhidas durante a laparoscopia, esse último considerado padrão-ouro para o diagnóstico da doença. Nos casos sem o resultado da laparoscopia, recomenda-se o tratamento empírico com base em exames secundários, como a ultrassonografia transvaginal, a ressonância magnética pélvica e os biomarcadores sanguíneos, dentre eles, o CA-125. ${ }^{20}$

Então, os exames de imagem têm papel dúbio no diagnóstico da endometriose, pois, apesar de serem peças fundamentais para confirmar casos suspeitos, não são sinais de certeza para a patologia. Quanto aos relatos em que, apesar de realizar os testes, não foi possível identificar a patologia, se atribui o fato de que muitas vezes as lesões são microscópicas, impedindo a visualização até mesmo por meio da laparoscopia. ${ }^{4}$

Independentemente do contexto geográfico, os profissionais enfrentam dificuldades similares e a endometriose é um problema de saúde pública que merece maior destaque e repercussão. Os resultados aqui apresentados apontam para a necessidade de uma melhor divulgação de informações sobre endometriose à população e aos profissionais de saúde. Por isso, necessitase de pesquisas futuras sobre intervenções para disseminar o conhecimento e sensibilizar a sociedade sobre essa situação bastante relevante, porém, amplamente negligenciada.

\section{CONSIDERAÇÕES FINAIS E IMPLICAÇÕES PARA A PRÁTICA}

As trajetórias das mulheres desde os primeiros sintomas na menarca até o diagnóstico definitivo de endometriose são permeadas por duas vertentes: as barreiras e os facilitadores. Apontam-se, como principais fatores dificultadores desse caminho, a desvalorização das queixas das pacientes por profissionais e pessoas do convívio, a naturalização da dor feminina, o impacto financeiro da doença e a dificuldade em estabelecer um diagnóstico diferencial. Por outro lado, as queixas de infertilidade, a capacidade individual de reconhecer a presença de uma patologia instaurada, o conhecimento sobre a endometriose e a experiência/capacitação do profissional permitem findar essas histórias.

Dentre as limitações deste estudo, ressalta-se a baixa abrangência de mulheres com diferentes perfis socioeconômicos. No entanto, isso reflete a realidade referente à dificuldade de acesso universal e à desigualdade social relacionadas ao diagnóstico de endometriose no Brasil, uma vez que a pesquisa estava disponível, via internet, para toda a população. Além disso, o caráter retrospectivo na coleta de dados pode interferir na memória das participantes ao contar suas próprias experiências, apesar de que todos os relatos analisados mantiveram uma coerência, compartilharam experiências semelhantes e puderam ser corroborados pela literatura científica.

Os resultados apoiam a realização de pesquisas futuras sobre a sensibilização da população geral acerca da endometriose e a construção de instrumentos que facilitem o diagnóstico precoce da patologia, como questionários de triagem específicos para dor pélvica crônica, escalas de avaliação da dor ginecológica e algoritmos de condução clínica.

No que tange a implicações para a prática, esta pesquisa contribui para o avanço científico por divulgar, de forma inédita, o caminho percorrido por mulheres até o diagnóstico clínico da endometriose no contexto dos serviços de saúde disponíveis 
no Brasil. Demonstraram-se os aspectos psicológicos e os impactos em diferentes níveis que podem ser acessados para criar experiências construtivas com relação à saúde da mulher. Além disso, reflexões sobre o papel da sociedade e dos profissionais podem ser levantadas por quem acessa esses resultados.

\section{CONTRIBUIÇÕES DOS AUTORES}

Desenho do estudo. Carla Marins Silva, Camilla Freitas da Cunha, Karoline Rangel Neves

Coleta ou produção dos dados. Carla Marins Silva, Camilla Freitas da Cunha, Karoline Rangel Neves

Análise de dados. Carla Marins Silva, Camilla Freitas da Cunha, Karoline Rangel Neves, Victor Hugo Alves Mascarenhas. Adriana Caroci-Becker

Interpretação dos resultados. Carla Marins Silva. Camilla Freitas da Cunha, Karoline Rangel Neves, Victor Hugo Alves Mascarenhas, Adriana Caroci-Becker.

Redação e revisão crítica do manuscrito. Carla Marins Silva, Camilla Freitas da Cunha, Karoline Rangel Neves, Victor Hugo Alves Mascarenhas, Adriana Caroci-Becker

Aprovação da versão final do artigo. Carla Marins Silva, Camilla Freitas da Cunha, Karoline Rangel Neves, Victor Hugo Alves Mascarenhas, Adriana Caroci-Becker

Responsabilidade por todos os aspectos do conteúdo e a integridade do artigo publicado. Carla Marins Silva, Camilla Freitas da Cunha, Karoline Rangel Neves, Victor Hugo Alves Mascarenhas, Adriana Caroci-Becker

\section{EDITOR ASSOCIADO}

Stela Maris de Mello Padoin (1)

\section{EDITOR CIENTÍFICO}

Ivone Evangelista Cabral (1)

\section{REFERÊNCIAS}

1. Hogg S, Vyas S. Endometriosis. Obstetrics, Gynaecol Reprod Med. 2015;25(5):133-41. http://dx.doi.org/10.1016/j.ogrm.2015.02.001.

2. Sousa TR, Queiroz AP, Baron RA, Sperandio Flores F. Prevalência dos sintomas da endometriose: revisão sistemática. CES Med [Internet]. 2015; [citado 2020 out 9];29(2):211-26. Disponível em: http://www.scielo.org.co/scielo.php?script=sci_arttext\&pid=S0120$87052015000200006 \&$ Ing $=$ en

3. National Institute for Health and Clinical Excellence. Endometriosis: diagnosis and management [Internet]. United Kingdom: NICE;2017 [citado 2020 out 9]. Disponível em: https://www.nice.org.uk/guidance/ng73

4. Ministério da Saúde (BR). Protocolos da atenção básica: saúde das mulheres [Internet]. Brasília: Instituto Sírio-Libanês de Ensino e Pesquisa; 2016. [citado 2020 out 9]. Disponível em: http://bvsms.saude.gov.br/bvs/

5. Roomaney R, Kagee A. Salient aspects of quality of life among women diagnosed with endometriosis: a qualitative study. J Health Psychol. 2018;23(7):905-16. http://dx.doi.org/10.1177/1359105316643069.

6. Brilhante AVM, Oliveira LAF, Lourinho LA, Manso AG. Narrativas autobiográficas de mulheres com endometriose: que fenômenos permeiam os atrasos no diagnóstico? Physis. 2019;29(3):e290307. http://dx.doi.org/10.1590/s0103-73312019290307.

7. Nnoaham KE, Hummelshoj L, Webster P, d'Hooghe T, Cicco Nardone F, Cicco Nardone $\mathrm{C}$ et al. Impact of endometriosis on quality of life and work productivity: a multicenter study across ten countries Fertil Steril. 2011;96(2):366-73.e8. http://dx.doi.org/10.1016/j. fertnstert.2011.05.090.

8. Greene R, Stratton P, Cleary SD, Ballweg ML, Sinaii N. Diagnostic experience among 4,334 women reporting surgically diagnosed endometriosis. Fertil Steril.2009;91(1):32-9. http://dx.doi.org/10.1016/j.fertnstert.2007.11.020.

9. van der Zanden M, Teunissen DAM, van der Woord IW, Braat DDM, Nelen WLDM, Nap AW. Barriers and facilitators to the timely diagnosis of endometriosis in primary care in the Netherlands. Fam Pract. 2020;37(1):131-6. http://dx.doi.org/10.1093/fampra/cmz041.

10. Andysz A, Jacukowicz A, Merecz-KotD, Najder A. Endometriosis: the challenge for occupational life of diagnosed women: a review of quantitative studies. Med Pr. 2018;69(6):663-71. http://dx.doi.org/10.13075/mp.5893.00737.

11. Minayo MCS. O desafio do conhecimento: pesquisa qualitativa em saúde. 14a ed. São Paulo: Hucitec; 2014.

12. Minayo MCS. Amostragem e saturação em pesquisa qualitativa: consensos e controvérsias. Rev Pesq Qualit [Internet]. 2017; [citado 2020 out 9];5(7):1-12. Disponível em: https://edisciplinas.usp.br/pluginfile. php/4111455/mod_resource/content/1/Minayosaturacao.pdf

13. Bardin L. Análise de conteúdo. Lisboa: Edições 70; 2009.

14. Leal MC, Pereira APE, Domingues RMSM, Theme Fa MM, Dias MAB, Nakamura-Pereira $\mathrm{M}$ et al. Obstetric interventions during labor and childbirth in Brazilian low-risk women. Cad Saude Publica. 2014;30(Sup 1):17-32. http://dx.doi.org/10.1590/0102-311X00151513.

15. Barros AJ, Santos IS, Matijasevich A, Domingues MR, Silveira M, Barros FC et al. Patterns of deliveries in a Brazilian birth cohort: almost universal cesarean sections for the better-off. Rev Saude Publica. 2011;45(4):63543. http://dx.doi.org/10.1590/S0034-89102011005000039.

16. Domingues RMSM, Dias MAB, Nakamura-Pereira M, Torres JA, d'Ors E, Pereira APE et al. Processo de decisão pelo tipo de parto no Brasil: da preferência inicial das mulheres à via de parto final. Cad Saude Publica. 2014;30(Supl 1):S101-16. http://dx.doi.org/10.1590/0102-311X00105113.

17. Zale M, Lambert E, LaNoue MD, Leader AE. Shedding light on endometriosis: Patient and provider perspectives on a challenging disease. J Endometr Pelvic Pain Disord. 2020;12(2):69-76. http://dx.doi. org/10.1177/2284026520905239.

18. American College Obstetricians and Gynecologists. Committee Opinion No 760: Dysmenorrhea and Endometriosis in the Adolescent. Obstet Gynecol. 2018;132(6):e249-58. http://dx.doi.org/10.1097/AOG.0000000000002978.

19. Gambadauro P, Carli V, Hadlaczky G. Depressive symptoms among women with endometriosis: a systematic review and meta-analysis. Am J Obstet Gynecol.2019;220(3):230-41. http://dx.doi.org/10.1016/j.ajog.2018.11.123.

20. Dunselman GA, Vermeulen N, Becker C, Calhaz-Jorge C, D'Hooghe T, De Bie B et al. European Society of Human Reproduction and Embryology (ESHRE) guideline: management of women with endometriosis. Hum Reprod. 2014;29(3):400-12. http://dx.doi.org/10.1093/humrep/det457.

21. Zondervan KT, Becker CM, Koga K, Missmer SA, Taylor RN, Viganò P. Endometriosis. Nat Rev Dis Primers. 2018;4(1):9. http://dx.doi. org/10.1038/s41572-018-0008-5.

22. Young K, Fisher J, Kirkman M. Women's experiences of endometriosis: a systematic review and synthesis of qualitative research.J Fam Plann Reprod Health Care.2015;41(3):225-34.http://dx.doi.org/10.1136/fprhc-2013-100853.

23. Marinho MCP, Magalhaes TF, Fernandes LFC, Augusto KL, Brilhante AVM, Bezerra LRPS. Quality of life in women with endometriosis: an integrative review. J Womens Health. 2018;27(3):399-408. http://dx.doi. org/10.1089/jwh.2017.6397.

24. Hennegan J, Shannon AK, Rubli J, Schwab KJ, Melendez-Torres GJ. Women's and girls' experiences of menstruation in low- and middle-income countries: a systematic review and qualitative metasynthesis. PLoS Med. 2019;16(5):e1002803. http://dx.doi.org/10.1371/journal.pmed.1002803.

25. Denouel A, Fauconnier A, Torre A. Attentes des femmes atteintes d'endométriose: quelle information apporter? RPC Endométriose CNGOFHAS [Expectations of women with endometriosis: what information to deliver? CNGOF-HAS Endometriosis Guidelines]. Gynécol Obstét Fertil Sénol. 2018;46(3):214-22.

26. Chauvet P, Guiguet-Auclair C, Comptour A, Denouël A, Gerbaud L, Canis $M$ et al. Feelings and expectations in endometriosis: analysis of open comments from a cohort of endometriosis patients. J Gynecol Obstet Hum Reprod.2018;47(7):281-7.http://dx.doi.org/10.1016/j.jogoh.2018.05.010. 